\title{
The first theoretical $\gamma$ Doradus instability strip
}

\author{
Anthony B. Kaye ${ }^{1,2}$, Phillip B. Warner ${ }^{3}$, Joyce A. Guzik ${ }^{4}$ \\ ${ }^{1}$ ITT Industries, Advanced Engineering and Sciences Division, 1551 \\ Wyoming Blvd., Kirtland Air Force Base, NM 87117, USA \\ ${ }^{2}$ Los Alamos National Laboratory, Applied Physics Division, MS F-663, \\ Los Alamos, NM 87545-2345, USA \\ ${ }^{3}$ National Optical Astronomy Observatory, 950 North Cherry Ave., \\ Tucson, AZ 85719, USA \\ ${ }^{4}$ Los Alamos National Laboratory, X-2, MS T085, Los Alamos, NM \\ 87545-2345, USA
}

\begin{abstract}
We present the first theoretical $\gamma$ Doradus instability strip. We find that our model instability strip agrees very well with the previously established, observationally based, instability strip of Handler \& Shobbrook (2002). We stress, as in Guzik et al. (2000), that the convection zone depth plays the major role in the determination of our instability strip. Once this depth becomes too deep or too shallow, the convection zone no longer allows for pulsational instability.
\end{abstract}

\section{Calculations and models}

The $\gamma$ Doradus stars are a recently-discovered class of main sequence pulsating variables lying near the red edge of the $\delta$ Scuti instability strip (Kaye et al. 1999). These stars pulsate in one to several modes with periods of 0.4 to $\sim 3 \mathrm{~d}$, believed to be gravity modes. We tested pulsational stability for approximately 15000 models lying in and around the $\delta$ Scuti instability strip, using the Pesnell (1990) linear non-adiabatic pulsation code, in the method described in Guzik et al. (2000). Our models have masses ranging from $1.00 \mathrm{M}_{\odot}$ to $2.50 \mathrm{M}_{\odot}$ in steps of $0.05 \mathrm{M}_{\odot}$, with ages ranging from $5 \times 10^{8}$ to $2 \times 10^{9} \mathrm{yr}$. Time steps in evolution range from $2 \mathrm{Myr}$ to $6 \mathrm{Myr}$.

We considered $\ell$ values of 1 to 5 , with periods ranging from 0.3 to $3 \mathrm{~d}$. We constrained our results to include only periods with growth rates $(\triangle \mathrm{KE} / \mathrm{KE}$ per period) greater than $10^{-7}$, to account for numerical uncertainties. We also considered models where the convective timescale at the base of the convection zone (CZ) is greater than or equal to the pulsation period, for validity of the frozen-in convection approximation in the driving region (Guzik 2000).

We present the first calculated $\gamma$ Dor instability strip, transposed into observational space, in Fig. 1. We also include the 30 bona fide $\gamma$ Dor stars, as presented in Table 2 in Warner, Kaye, \& Guzik (2003). A theoretical plot of the calculations can also be found in that paper. This instability strip is bounded by $\sim 6850-7360 \mathrm{~K}$ at the red and blue edge, respectively, on the zero-age main 


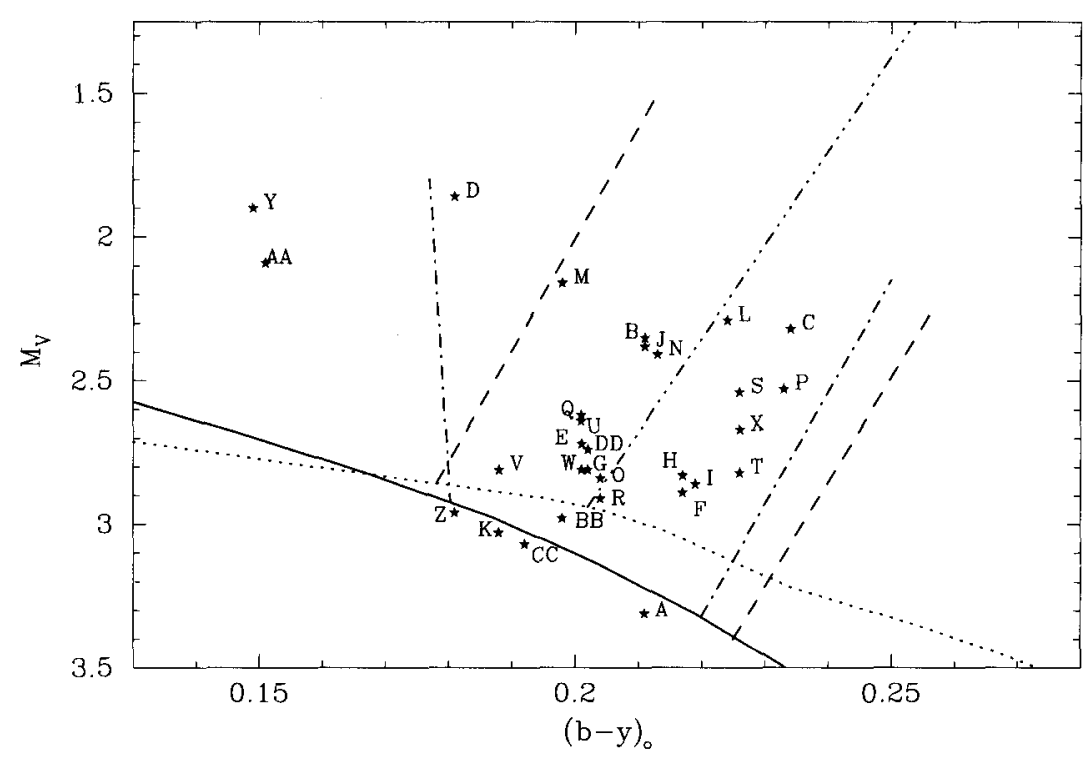

Figure 1. Extreme edges of the calculated instability strip (dashed lines) and calculated main sequence (solid line) compared with the observational red edge of the $\delta$ Scuti instability strip (Rodriguez \& Breger 2001; triple dot-dash line) and $\gamma$ Dor instability strip (Handler \& Shobbrook 2002; dot-dash line). Also shown is the observed Crawford main sequence (1975; dotted line) and all 30 bona fide $\gamma$ Dor stars (see Table 2 in Warner, Kaye, \& Guzik 2003).

sequence and by $\sim 6560-7000 \mathrm{~K}$ at the red and blue edge, respectively, approximately two magnitudes more luminous.

The CZ depth for three models, $\mathrm{M}=1.45,1.55$, and $1.75 \mathrm{M}_{\odot}$, is shown in Fig. 2. The $\gamma$ Dor phenomenon is dependent upon the depth of the CZ, as proposed in Guzik et al. (2000). As the bottom of the CZ enters the range between $\sim 0.91$ to $\sim 0.96 \mathrm{R}_{\star}$, the $\mathrm{CZ}$ is sufficiently deep to sustain $\gamma$ Dor-type pulsations. As the model CZ becomes shallower, the convective timescale becomes shorter than the pulsation period, and convection can adapt rapidly enough to transport the emerging luminosity, and therefore quench the pulsation. As the depth of the $\mathrm{CZ}$ becomes deeper in our models, the $\mathrm{CZ}$ becomes deep enough for radiative damping to dominate the pulsation driving that occurs at the base of the envelope CZ.

We also present the roughly Gaussian shape of the instability strip (Fig. 3). The tail on the red edge occurs as the convection zone becomes too deep, while the tail on the blue edge occurs as the convection zone disappears. The largest growth rates lie in the center of the strip, where we find most $\gamma$ Dor stars observationally. The slope of each extreme, and thus of the entire instability strip, is along a line of constant convective turnover time, again showing the crucial role that convection plays in the physics of these stars. 


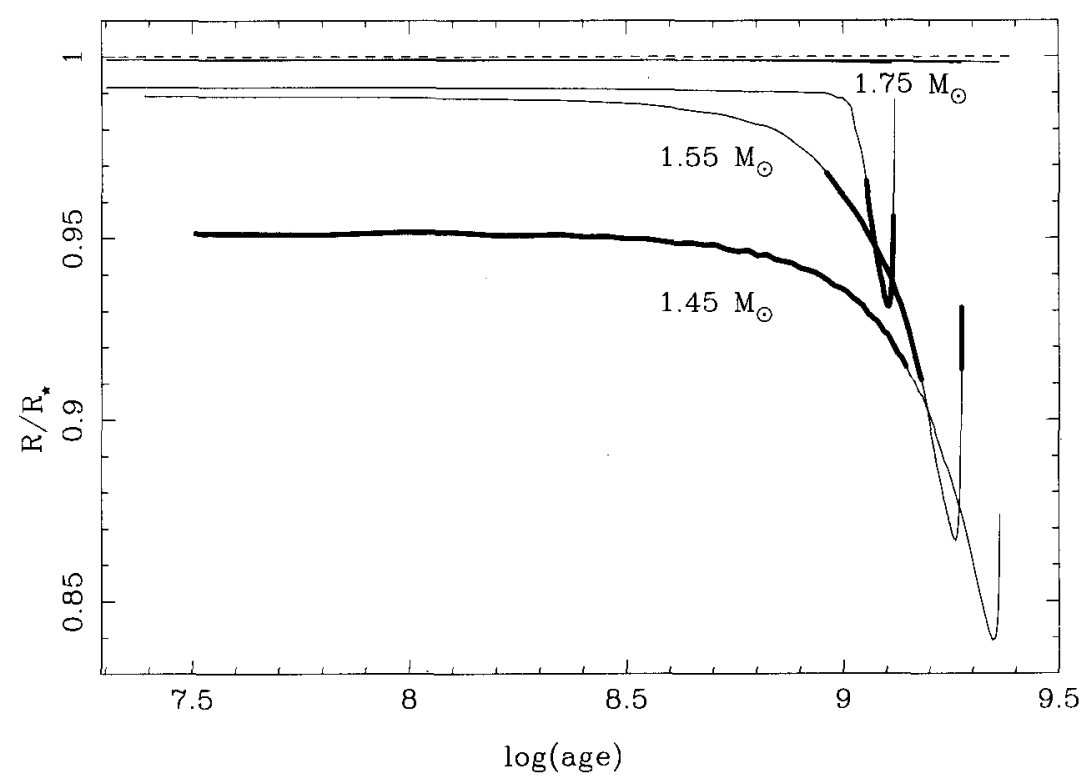

Figure 2. Convection zone depth for $\mathrm{M}=1.45,1.55$, and $1.75 \mathrm{M}_{\odot}$ for $\ell=1$. The dashed line is the normalized radius of the model; the thin solid line just below the normalized radius is the top of the convection zone for each model. Thin solid lines represent the base of the convection zone; thick solid lines indicate where models exhibited $\gamma$ Doradus-type pulsations.

\section{References}

Crawford, D.L. 1975, AJ, 80, 955

Guzik, J.A., Kaye, A.B., Bradley, P.A., Cox, A.N., Neuforge, C. 2000, ApJ, 542, L57

Handler, G., Shobbrook, R.R. 2002, MNRAS, 333, 251

Kaye, A.B., Handler, G., Krisciunas, K., Poretti, E., Zerbi, F.M. 1999, PASP, 111,840

Pesnell, W.D. 1990, ApJ, 363, 227

Rodríguez, E., Breger, M. 2001, A\&A, 366, 178

Warner, P.B., Kaye, A.B., Guzik, J.A. 2003, ApJ, 593, 1049 


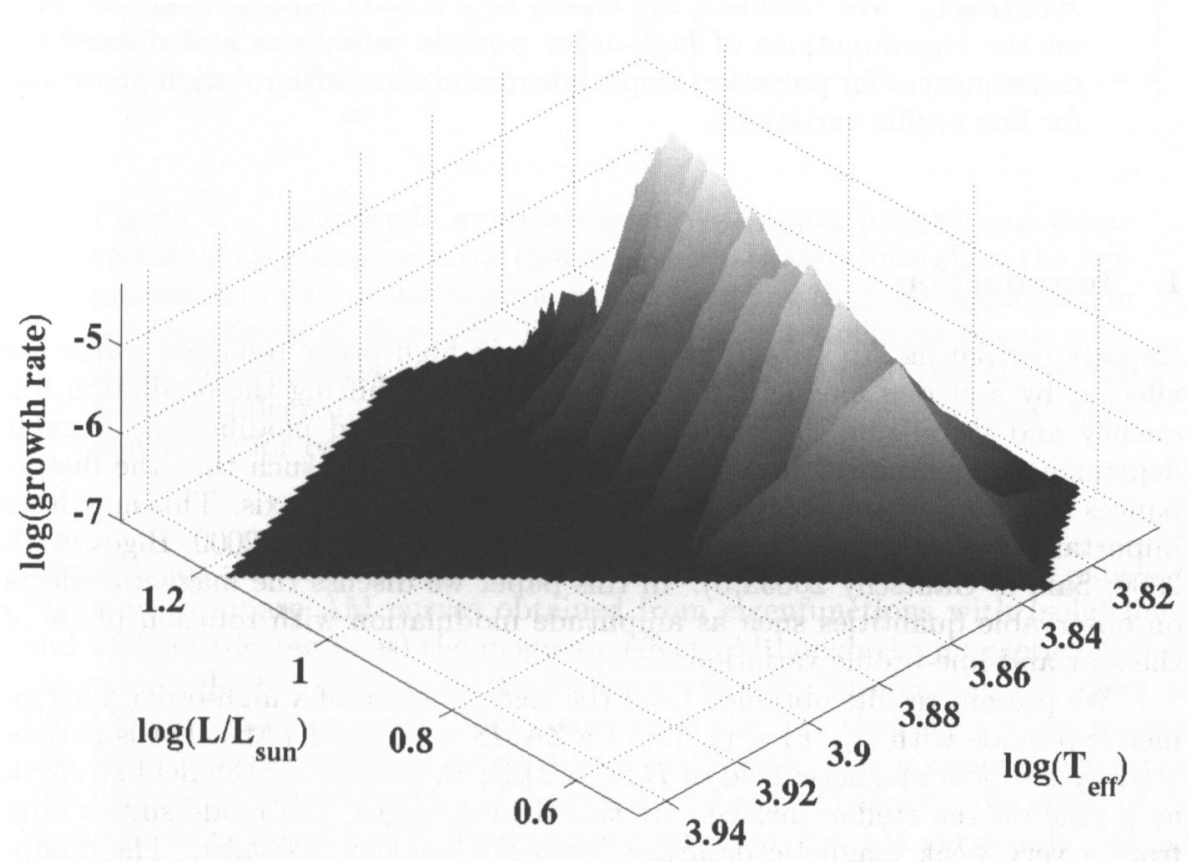

Figure 3. Plot of growth rates of models in our calculated instability strip for $\ell=1$ in the $T_{\text {eff }}-L$ plane. This is the lower bounding value of $\ell$, representing the most restrictive case. The log of the growth rate ( $\triangle \mathrm{KE} / \mathrm{KE}$ per period) is shown on the $z$-axis. 